\title{
An approach to source characterization of tremor signals associated with eruptions and lahars
}

\author{
Hiroyuki Kumagai ${ }^{1 *}$, Patricia Mothes ${ }^{2}$, Mario Ruiz ${ }^{2}$ and Yuta Maeda ${ }^{1}$
}

\begin{abstract}
Tremor signals are observed in association with eruption activity and lahar descents. Reduced displacement $\left(D_{R}\right)$ derived from tremor signals has been used to quantify tremor sources. However, tremor duration is not considered in $D_{R}$, which makes it difficult to compare $D_{R}$ values estimated for different tremor episodes. We propose application of the amplitude source location (ASL) method to characterize the sources of tremor signals. We used this method to estimate the tremor source location and source amplitude from high-frequency $(5-10 \mathrm{~Hz})$ seismic amplitudes under the assumption of isotropic S-wave radiation. We considered the source amplitude to be the maximum value during tremor. We estimated the cumulative source amplitude $\left(I_{s}\right)$ as the offset value of the timeintegrated envelope of the vertical seismogram of tremor corrected for geometrical spreading and medium attenuation in the $5-10-\mathrm{Hz}$ band. For eruption tremor signals, we also estimated the cumulative source pressure $\left(I_{\mathrm{p}}\right)$ from an infrasonic envelope waveform corrected for geometrical spreading. We studied these parameters of tremor signals associated with eruptions and lahars and explosion events at Tungurahua volcano, Ecuador. We identified two types of eruption tremor at Tungurahua: noise-like inharmonic waveforms and harmonic oscillatory signals. We found that $I_{s}$ increased linearly with increasing source amplitude for lahar tremor signals and explosion events, but $I_{s}$ increased exponentially with increasing source amplitude for inharmonic eruption tremor signals. The source characteristics of harmonic eruption tremor signals differed from those of inharmonic tremor signals. We found a linear relation between $I_{s}$ and $I_{p}$ for both explosion events and eruption tremor. Because $I_{p}$ may be proportional to the total mass involved during an eruption episode, this linear relation suggests that $I_{s}$ may be useful to quantify eruption size. The $I_{s}$ values we estimated for inharmonic eruption tremor were consistent with previous estimates of volumes of tephra fallout. The scaling relations among source parameters that we identified will contribute to our understanding of the dynamic processes associated with eruptions and lahars. This new approach is applicable in analyzing tremor sources in real time and may contribute to early assessment of the size of eruptions and lahars.
\end{abstract}

Keywords: Amplitude source location method, High-frequency seismic amplitude, Scaling relation, Ejecta volume, Volcano monitoring

\section{Findings}

Introduction

Volcanic eruptions and lahars excite tremor signals that exhibit oscillatory and/or noise-like waveforms lasting for periods of several tens of seconds to hours or longer. Quantifying the sources of these signals is important for understanding the dynamic processes associated with

\footnotetext{
*Correspondence: kumagai@eps.nagoya-u.ac.jp

'Graduate School of Environmental Studies, Nagoya University, Furo-cho,

Chikusa-ku, Nagoya 464-8601, Japan

Full list of author information is available at the end of the article
}

eruptions and lahars and for monitoring volcanic activity. Reduced displacement $\left(D_{\mathrm{R}}\right)$, a measurable quantity defined by Aki and Koyanagi (1981), has been used in various studies of tremor signals (e.g., Fehler 1983; McNutt 1992; Alparone et al. 2003; Benoit et al. 2003). McNutt (1994) showed that the volcanic explosivity index (VEI; Newhall and Self 1982) is correlated with $D_{\mathrm{R}}$ estimated for eruption tremor, although McNutt (2004) later showed that there is a wide range of $D_{\mathrm{R}}$ values for each VEI. McNutt and Nishimura (2008) investigated the relations between $D_{\mathrm{R}}$ and several factors such as vent

\section{实 Springer}


radius, erupted volume, and tremor time history. There are, however, fundamental problems in using $D_{\mathrm{R}}$ to characterize tremor sources. Aki and Koyanagi (1981) originally defined $D_{\mathrm{R}}$ as the root-mean-square displacement amplitude corrected for geometrical spreading for each spectral peak in tremor and showed from observed tremor signals that $D_{\mathrm{R}}$ depends on tremor's frequency. Furthermore, tremor duration is not considered in the calculation of $D_{\mathrm{R}}$. These issues complicate making comparison of $D_{\mathrm{R}}$ values estimated for tremor episodes having different tremor frequencies and durations.

Battaglia et al. (2005a, b) showed that a source location method using seismic amplitudes is useful for locating the source of tremor signals associated with eruptions at the Piton de la Fournaise volcano, and they used the tremor source amplitude to estimate the amount of erupted lava. Kumagai et al. (2013) demonstrated that the source amplitude determined by the amplitude source location (ASL) method in a frequency band of $5-10 \mathrm{~Hz}$ can be used as a quantitative measure of the size of various volcano-seismic events. In this study, we extended the methodology of the above studies to estimate the source amplitudes and cumulative source amplitudes for individual tremor signals associated with eruptions and lahars and explosion events at Tungurahua volcano, Ecuador. There are two main types of eruption tremor at Tungurahua: noise-like inharmonic waveforms and harmonic oscillations. Both types of tremor exhibit scaling relations, which are useful for characterization of the sources of tremor signals. We show that inharmonic and harmonic tremors have different source characteristics and that the cumulative source amplitudes of inharmonic tremor are consistent with previous estimates of ejecta volume. These parameters may be used to measure tremor signals and derive estimates of the size of volcanic eruptions and lahars.

\section{Method}

Battaglia and Aki (2003) developed the ASL method, which uses seismic amplitudes under the assumption of isotropic $S$-wave radiation. Kumagai et al. (2010) showed that this method can be used to locate various volcano-seismic signals, including tremor associated with eruptions and lahars in a high-frequency band, where the assumption of isotropic $S$-wave radiation is valid because of the path effect caused by scattering of seismic waves (Takemura et al. 2009; Kumagai et al. 2011a). The ASL method has been used in various volcano-seismic studies (e.g., Battaglia et al. 2003, 2005a, 2005b; Kumagai et al. 2011b, 2014; Ogiso and Yomogida 2012, 2015). Kumagai et al. (2013) estimated the source amplitudes of volcano-tectonic earthquakes, long-period events, and explosion events using the ASL method and found the following scaling relation between seismic magnitude $(M)$ and source amplitude $\left(A_{\mathrm{s}}\right)$ :

$$
M=1.10 \log A_{\mathrm{s}}+2.96 .
$$

In this relation, $M$ was derived from the relation of Watanabe (1971) using maximum vertical velocity amplitudes and $A_{\mathrm{s}}\left(\mathrm{m}^{2} / \mathrm{s}\right)$ was estimated from vertical velocity envelopes corrected for geometrical spreading and medium attenuation in the $5-10-\mathrm{Hz}$ frequency band (see below). This relation indicates that source amplitudes estimated in the high-frequency band can be used to quantify event size. In the following, we extend the approaches described above to quantify tremor sources.

We follow the ASL method of Kumagai et al. (2010, 2013), in which grid nodes are distributed in a search domain and the far-field $S$-wave approximation is used. Since we focus on tremor signals associated with eruptions and lahars, grid nodes are positioned over the volcano surface. The particle velocity $v_{i j}$ band-passed in frequency around $f$ at the $i$ th station from the $j$ th grid node may be written as

$$
v_{i j}\left(t_{s}+\tau_{i j}\right)=A_{0}\left(\boldsymbol{\xi}_{j}\right) s_{0}\left(t_{s}\right) \frac{1}{r_{i j}} e^{-C \tau_{i j}},
$$

where $t_{\mathrm{s}}$ is the source origin time, $r_{i j}$ is the distance between station $i$ and source node $j, \tau_{i j}$ is the $S$-wave travel time from the $j$ th grid node to the $i$ th station, $\boldsymbol{\xi}_{j}$ is the location of the $j$ th grid node, and $A_{0}$ and $s_{0}$ are the radiation coefficient and the second time derivative of the moment function, respectively. We assume a constant value of $A_{0}$, which corresponds to isotropic $S$-wave radiation. Here, $C$ is given by

$$
C=\frac{\pi f}{Q},
$$

where $Q$ is the quality factor of an $S$ wave for medium attenuation. We use sliding time windows for the source time $t_{s}^{k}$, defined as

$$
t_{s}^{k}=t_{s}+(k-1) T_{\mathrm{w}}, \quad(k=1,2,3, \ldots)
$$

where $T_{\mathrm{w}}$ is the window duration. The envelope amplitude averaged over the time window $T_{\mathrm{w}}$ is obtained from eqs. 2 and 4 as follows:

$$
\begin{aligned}
g_{i j}\left(t_{s}^{k}+\tau_{i j}\right) & =\frac{1}{T_{w}} \int_{0}^{T_{w}} \tilde{v}_{i j}\left(t+t_{s}^{k}+\tau_{i j}\right) d t, \\
& =A_{j k} \frac{1}{r_{i j}} e^{-C \tau_{i j}},
\end{aligned}
$$

where $\tilde{v}_{i j}(t)$ is the envelope of $v_{i j}(t)$. Here, $A_{j k}$, which is the source amplitude at the $j$ th grid node and source time $t_{s}^{k}$, is given as 

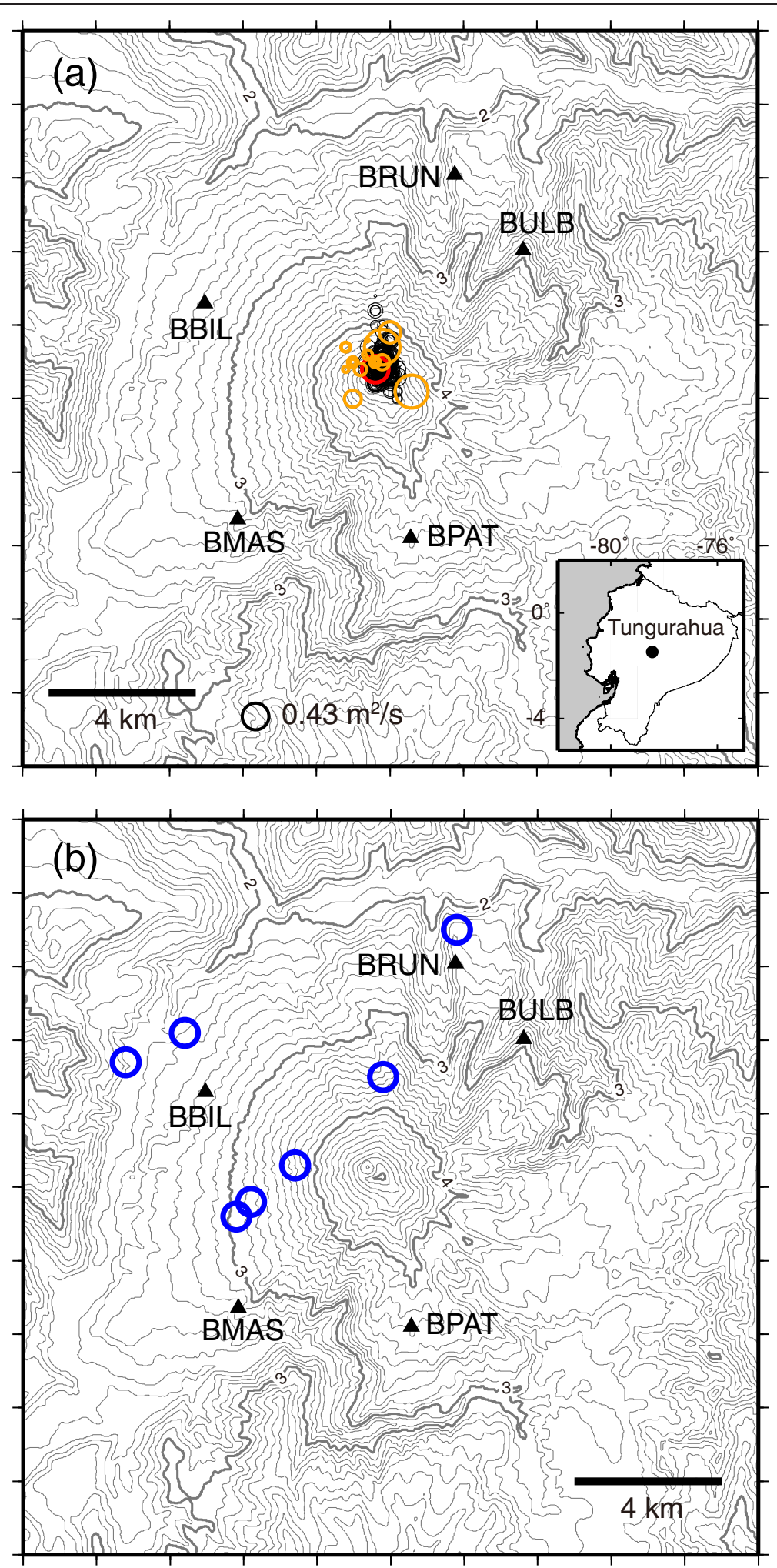

Fig. 1 Source locations of eruption and lahar tremor signals. a Observation station locations (solid triangles) at Tungurahua volcano, Ecuador. Black and red circles represent source locations and amplitudes, respectively, of an eruption tremor episode on 16-17 March 2013 (Fig. 2). Orange circles indicate source locations and amplitudes of other eruption tremor episodes listed in Table 1. The radius of each circle indicates the source amplitude scaled to the reference circle at the lower left of the map. Topographic contour interval is $100 \mathrm{~m}$. Inset map shows the location of Tungurahua volcano. b Source locations of lahar tremor signals listed in Table 2 (blue circles) 


$$
A_{j k}=\frac{1}{T_{\mathrm{w}}} A_{0}\left(\boldsymbol{\xi}_{j}\right) \int_{0}^{T_{\mathrm{w}}} \tilde{s}_{0}\left(t+t_{s}^{k}\right) d t
$$

where $\tilde{s}_{0}(t)$ is the envelope of $s_{0}(t)$. We estimate the source amplitude $A_{j k}$ by using the following equation:

$$
A_{j k}=\frac{1}{N} \sum_{i=1}^{N} g_{i}^{o}\left(t_{s}^{k}+\tau_{i j}\right) r_{i j} e^{C \tau_{i j}}
$$

where $N$ is the number of stations. Here, $g_{i}^{o}(t)$ is the observed velocity envelope amplitude averaged over the time window $T_{\mathrm{w}}$ at the $i$ th station:

$$
g_{i}^{o}\left(t_{s}^{k}+\tau_{i j}\right)=\frac{1}{T_{w}} \int_{0}^{T_{w}} \tilde{v}_{i}^{o}\left(t+t_{s}^{k}+\tau_{i j}\right) d t
$$

where $\tilde{v}_{i}^{o}(t)$ is the envelope of the observed velocity waveform $v_{i}^{o}(t)$ corrected for site amplification as a function of $f$. To evaluate the fits between the observed and calculated amplitudes in each time window, we use the normalized residual function $E_{j k}$, defined as

$$
E_{j k}=\frac{\sum_{i=1}^{N}\left\{g_{i}^{o}\left(t_{s}{ }^{k}+\tau_{i j}\right)-g_{i j}\left(t_{s}{ }^{k}+\tau_{i j}\right)\right\}^{2}}{\sum_{i=1}^{N}\left\{g_{i}^{o}\left(t_{s}{ }^{k}+\tau_{i j}\right)\right\}^{2}} .
$$

We use a grid search to find the minimum residual between the observed and calculated amplitudes in each time window. We identify the maximum $A_{j k}$ among those
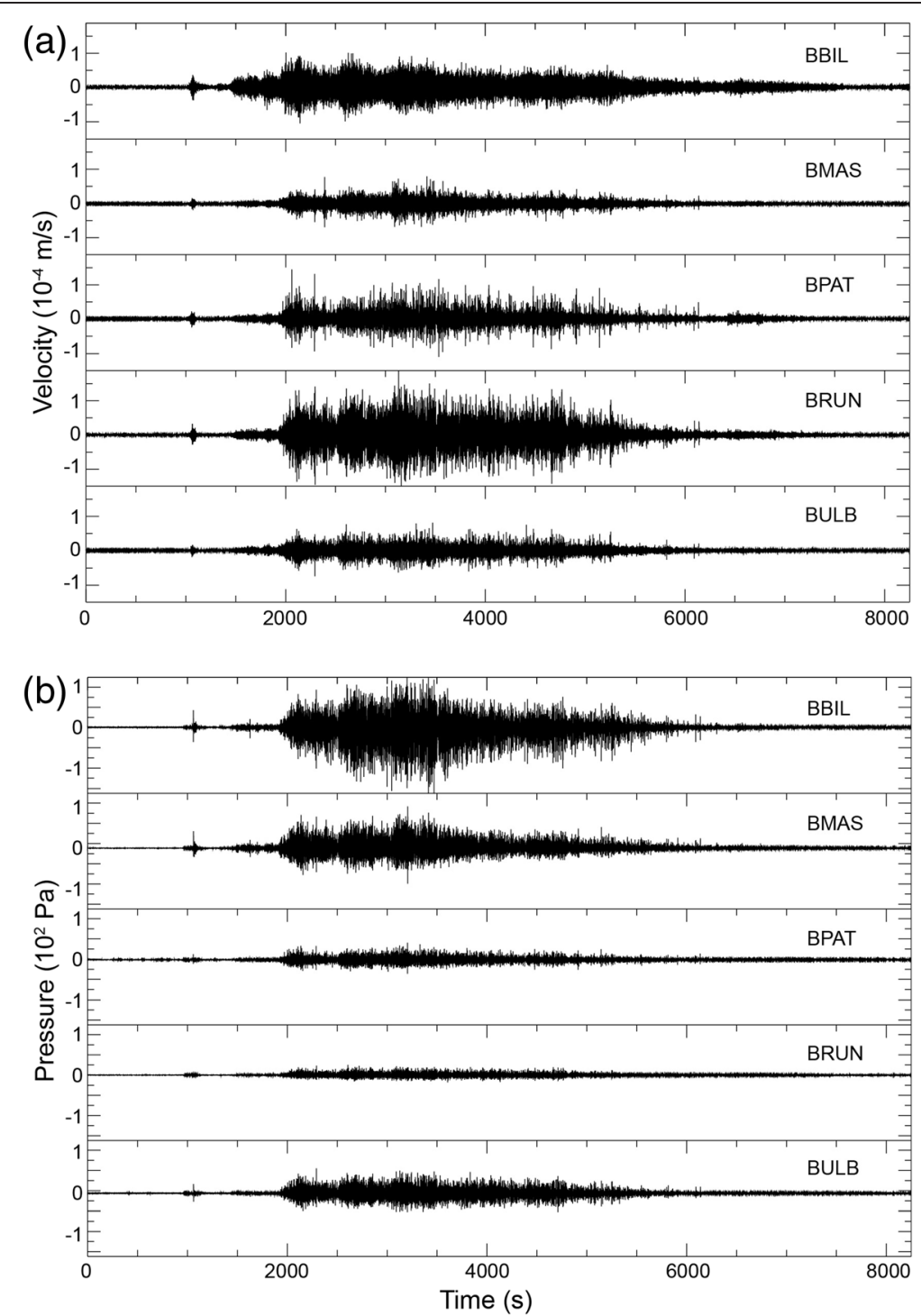

Fig. 2 Eruption tremor records. Seismic (a) and infrasonic (b) records from the five stations shown in Fig. 1 during the eruption episode on 16-17 March 2013 
estimated at the minimum residual positions in individual sliding time windows during tremor. We regard this maximum $A_{j k}$ and its position as the source amplitude $\left(A_{s}\right)$ and location of tremor, respectively.

We define the cumulative source amplitude $\left(I_{s}\right)$ as

$$
I_{s}=\frac{1}{N} \sum_{i=1}^{N} \int_{0}^{T} \tilde{v}_{i}^{o}(t) r_{i} e^{C \tau_{i}} d t
$$

where $T$ is the tremor duration, $r_{i}$ is the distance between the source and the $i$ th station, and $\tau_{i}$ is the $S$-wave travel time from the source to the $i$ th station. Here, we call $\tilde{v}_{i}^{o}(t) r_{i} e^{C \tau_{i}}$ the source amplitude function. For eruption tremor, we also define the cumulative source pressure $\left(I_{\mathrm{p}}\right)$ as

$$
I_{p}=\frac{1}{N} \sum_{i=1}^{N} \int_{0}^{T} \tilde{P}_{i}(t) r_{i} d t
$$

where $\tilde{P}_{i}(t)$ is the envelope of the observed infrasonic waveform. We also estimate reduced displacement $\left(D_{\mathrm{R}}\right)$ defined as

$$
D_{R}=\frac{1}{N} \sum_{i=1}^{N} a_{i} r_{i} /(2 \sqrt{2})
$$

where $a_{i}$ is the peak-to-peak seismic amplitude of tremor at the $i$ th station.

\section{Results}

\section{Tremor signals}

Tungurahua (elevation, $5023 \mathrm{~m}$ ) is an andesitic stratovolcano in the Ecuadorian Andes. This volcano has continued its eruptive activity since 1999, in which major eruptions were characterized by vulcanian and strombolian eruptive styles and were accompanied by pyroclastic flows (Hall et al. 1999, 2013; Samaniego et al. 2011; Mothes et al. 2015). There are five permanent broadband seismic stations at Tungurahua (Fig. 1a), which were installed by an international collaboration between Japan and Ecuador (Kumagai et al. 2010). Each station is equipped with a broadband seismometer (Güralp CMG$40 \mathrm{~T}, 0.02-60 \mathrm{~s}$ ) and an infrasonic sensor (ACO 7144/ 4144, 0.01-10 s).

We performed ASL analysis of tremor signals associated with eruptions and lahars recorded by this network. Following Kumagai et al. (2010, 2013), we used filtered (5-10-Hz band-pass) vertical velocity seismograms. We did not correct for site amplification factors because Kumagai et al. (2010) reported that the normalized residuals for an explosion event at Tungurahua were smaller without the corrections. We used a 10-s sliding time window with no overlap, grid intervals of $200 \mathrm{~m}$, a homogeneous $S$-wave velocity of $1443 \mathrm{~m} / \mathrm{s}$, and $Q=60$
(Kumagai et al. 2010). Coordinated universal time (UTC) was used throughout this study.

Figure 2a, b shows seismic and infrasonic records, respectively, at the five stations during an eruption episode on 16-17 March 2013. This episode started with a vulcanian outbreak followed by sustained eruptions for more than $1 \mathrm{~h}$. There are two types of tremor associated with eruption activity at Tungurahua: noise-like inharmonic seismic waveforms and repetitive
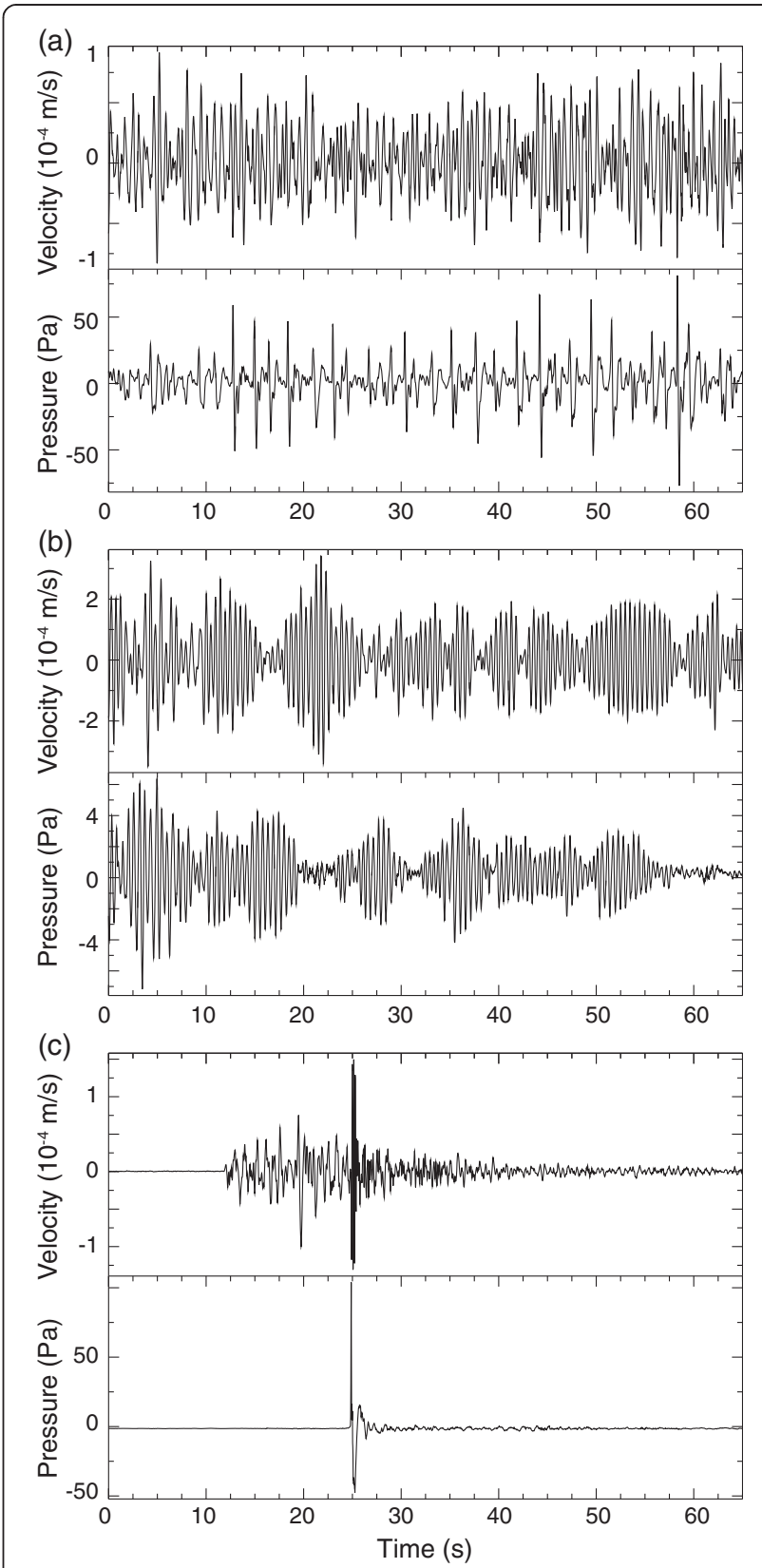

Fig. 3 Eruption tremor signals and an eruption event. Vertical seismic and infrasonic records of $\mathbf{a}$ inharmonic tremor on 16 March 2013 at station BBIL, b harmonic tremor on 2 February 2014 at station BBIL, and $\mathbf{c}$ an explosion event on 7 December 2011 at station BPAT 


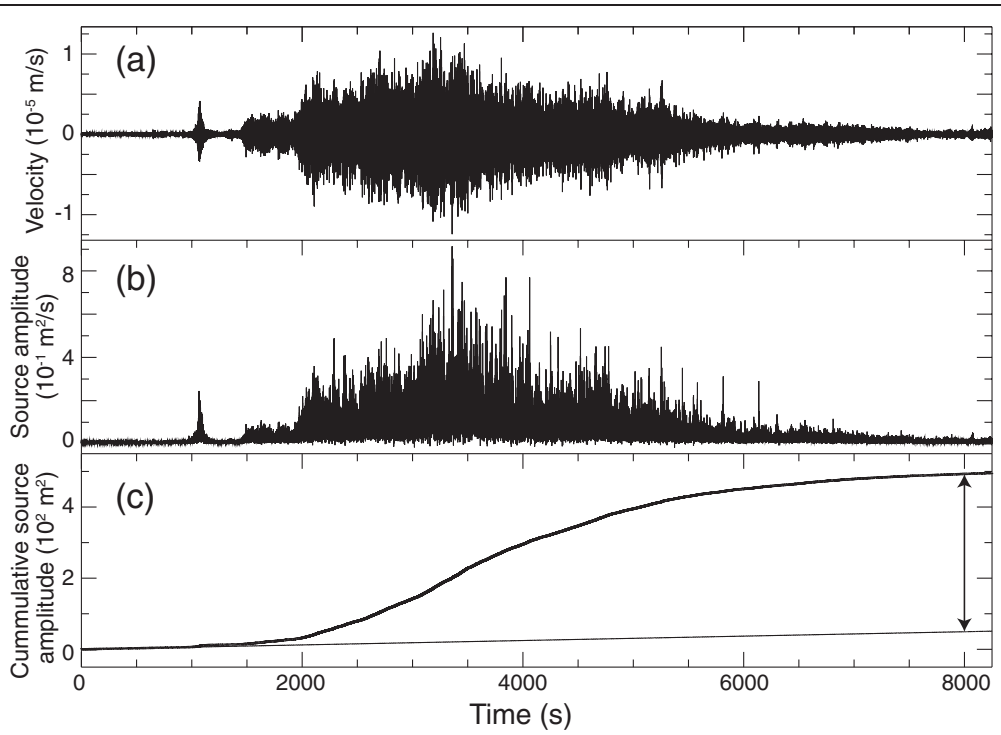

Fig. 4 Cumulative source amplitude estimation. a Band-pass-filtered $(5-10 \mathrm{~Hz})$ vertical velocity seismogram recorded at station BBIL during the eruption episode on 16-17 March 2013. b Envelope of seismogram in a corrected for geometrical spreading and medium attenuation (source amplitude function). c Time integration of seismogram in $\mathbf{b}$ (thick line) and the linear trend estimated from pre-tremor noise (thin line). Offset value from trend line at the end of the tremor (arrows) was regarded as the cumulative source amplitude

infrasonic impulses (Fig. 3a), which we call inharmonic tremor, and harmonic oscillations in both seismic and infrasonic waveforms with dominant peak frequencies around $1-2 \mathrm{~Hz}$ (Fig. 3b), which we call harmonic tremor. We note that harmonic tremor contained seismic energies in $5-10 \mathrm{~Hz}$, although the peaks existed around $1-2 \mathrm{~Hz}$. Inharmonic and harmonic tremors have been observed at various volcanoes (e.g., McNutt 1992; Garcés et al. 1998; Johnson 2003; Lees et al. 2008; Matsumoto et al. 2013; Ichihara et al. 2013).

Table 1 Eruption tremor episodes analyzed in this study. Tremor duration was estimated as the interval during which the envelope amplitude averaged over a 5-s interval was larger than the noise amplitude before the tremor onset

\begin{tabular}{|c|c|c|c|c|}
\hline Date and time of tremor onset & Duration (s) & Source amplitude $\left(\mathrm{m}^{2} / \mathrm{s}\right)$ & Cumulative source amplitude $\left(\mathrm{m}^{2}\right)$ & Cumulative source pressure $(\mathrm{Pa} \mathrm{m} \mathrm{s})$ \\
\hline \multicolumn{5}{|l|}{ Inharmonic tremor } \\
\hline 2010-05-28 13:46:53 & 28380 & $(5.3 \pm 1.0) \times 10^{-1}$ & $(3.71 \pm 0.43) \times 10^{3}$ & $(1.19 \pm 0.30) \times 10^{9}$ \\
\hline 2012-12-16 12:34:45 & 9345 & $(2.49 \pm 0.46) \times 10^{-1}$ & $(8.20 \pm 0.12) \times 10^{2}$ & $(2.67 \pm 0.57) \times 10^{8}$ \\
\hline 2012-12-16 15:10:05 & 24350 & $(3.45 \pm 0.21) \times 10^{-1}$ & $(1.84 \pm 0.14) \times 10^{3}$ & $(5.0 \pm 1.0) \times 10^{8}$ \\
\hline 2013-03-16 22:56:57 & 7620 & $(4.26 \pm 0.59) \times 10^{-1}$ & $(7.68 \pm 0.90) \times 10^{2}$ & $(1.83 \pm 0.35) \times 10^{8}$ \\
\hline 2013-07-14 11:46:32 & 3895 & $(2.81 \pm 0.13) \times 10^{-1}$ & $(5.6 \pm 1.1) \times 10^{2}$ & $(1.08 \pm 0.18) \times 10^{8}$ \\
\hline 2014-02-01 22:38:46 & 17795 & $(5.76 \pm 0.78) \times 10^{-1}$ & $(2.05 \pm 0.29) \times 10^{3}$ & $(6.4 \pm 1.4) \times 10^{8}$ \\
\hline \multicolumn{5}{|l|}{ Harmonic tremor } \\
\hline 2010-05-29 12:54:41 & 235 & $(9.44 \pm 0.47) \times 10^{-2}$ & $(1.16 \pm 0.09) \times 10^{1}$ & $(2.66 \pm 0.33) \times 10^{6}$ \\
\hline 2010-05-29 21:07:21 & 420 & $(1.96 \pm 0.26) \times 10^{-1}$ & $(2.61 \pm 0.12) \times 10^{1}$ & $(5.15 \pm 0.96) \times 10^{6}$ \\
\hline 2010-05-29 21:15:07 & 125 & $(5.70 \pm 0.82) \times 10^{-2}$ & $2.84 \pm 0.45$ & $(2.0 \pm 1.0) \times 10^{5}$ \\
\hline 2010-05-29 23:51:24 & 260 & $(1.12 \pm 0.13) \times 10^{-1}$ & $(1.25 \pm 0.14) \times 10^{1}$ & $(2.67 \pm 0.48) \times 10^{6}$ \\
\hline 2012-12-17 03:45:42 & 690 & $(1.70 \pm 0.14) \times 10^{-1}$ & $(3.31 \pm 0.44) \times 10^{1}$ & $(1.74 \pm 0.36) \times 10^{6}$ \\
\hline 2012-12-17 05:36:10 & 2605 & $(1.55 \pm 0.15) \times 10^{-1}$ & $(1.63 \pm 0.13) \times 10^{2}$ & $(2.27 \pm 0.46) \times 10^{7}$ \\
\hline 2012-12-17 08:16:46 & 965 & $(1.76 \pm 0.18) \times 10^{-1}$ & $(1.04 \pm 0.11) \times 10^{2}$ & $(1.23 \pm 0.29) \times 10^{7}$ \\
\hline 2014-02-02 02:34:17 & 145 & $(1.06 \pm 0.73) \times 10^{-1}$ & $6.86 \pm 0.82$ & $(4.8 \pm 1.7) \times 10^{5}$ \\
\hline 2014-02-02 12:24:28 & 510 & $(1.50 \pm 0.89) \times 10^{-1}$ & $(4.05 \pm 0.43) \times 10^{1}$ & $(1.54 \pm 0.30) \times 10^{6}$ \\
\hline 2014-02-02 21:55:24 & 875 & $(1.59 \pm 0.40) \times 10^{-1}$ & $(5.97 \pm 0.40) \times 10^{1}$ & $(1.38 \pm 0.23) \times 10^{7}$ \\
\hline
\end{tabular}




\section{ASL analysis}

For our ASL analysis of the tremor shown in Fig. 2, we used large amplitude portions of the tremor seismic signals. ASLs for individual time windows were determined to be near the summit vent (black circles in Fig. 1a), where the maximum source amplitude and its location (red circle in Fig. 1a) were regarded as the source amplitude and location for this tremor. We then estimated the cumulative source amplitude $\left(I_{\mathrm{s}}\right)$ and pressure $\left(I_{\mathrm{p}}\right)$. To estimate $I_{\mathrm{s}}$, we used the envelope of the vertical velocity seismogram in the $5-10 \mathrm{~Hz}$ frequency band (Fig. 4a, b). We first estimated the linear trend of the pre-tremor noise level and then measured the offset value from the trend line at the end of the tremor (Fig. 4c). We used the seismic waveforms at different stations for which the offsets were clearly defined and averaged the offset values to estimate $I_{\mathrm{s}}$ for each tremor episode. We estimated $I_{\mathrm{p}}$ by the same method but using the unprocessed infrasonic waveforms. We estimated $D_{\mathrm{R}}$ using vertical seismograms with a $1-\mathrm{Hz}$ high-pass filter.

The above method was used to analyze tremor signals associated with eruption episodes between 2010 and 2014 (Table 1); these included a number of harmonic tremor signals, from which we selected their typical signals for our analyses. ASLs for both the inharmonic and harmonic tremor signals were determined to be near the summit vent (orange circles in Fig. 1a).

We also estimated ASLs, source amplitudes, $I_{\mathrm{s}}, I_{\mathrm{p}}$, and $D_{\mathrm{R}}$ values for explosion events reported by Kumagai et al. (2013). Following the procedure of Kumagai et al. (2013), we used time windows that preceded the arrivals of infrasonic waves to estimate source amplitudes and locations because the seismograms were strongly affected by seismic waves generated by the infrasonic waves (Fig. 3c). ASLs for these events were also determined to be near the summit vent. We used the total seismogram to estimate the $I_{\mathrm{s}}$ value for each explosion event, so the results included the effects of infrasonic waves.

Our plots of $I_{\mathrm{s}}$ values versus source amplitudes (Fig. 5a) indicate that $I_{\mathrm{s}}$ increased linearly with source amplitude for the explosion events, but $I_{\mathrm{s}}$ increased exponentially with the source amplitude for the inharmonic tremor signals. The harmonic tremor signals had similar source amplitudes to those of the explosion events and $I_{\mathrm{s}}$ values that lay between those of the explosion events and the inharmonic tremor signals. We did not find a clear relation between $I_{\mathrm{s}}$ and source amplitude for the harmonic tremor signals. There was a proportional relation between $I_{\mathrm{p}}$ and $I_{\mathrm{s}}$ for the tremor signals and explosion events (Fig. 5b) but no clear proportional relation between $I_{\mathrm{p}}$ and $D_{\mathrm{R}}$, especially for tremor signals (Fig. 5c).

Tremor signals associated with lahars at Tungurahua on 17 February 2011 (Fig. 6) were characterized by long
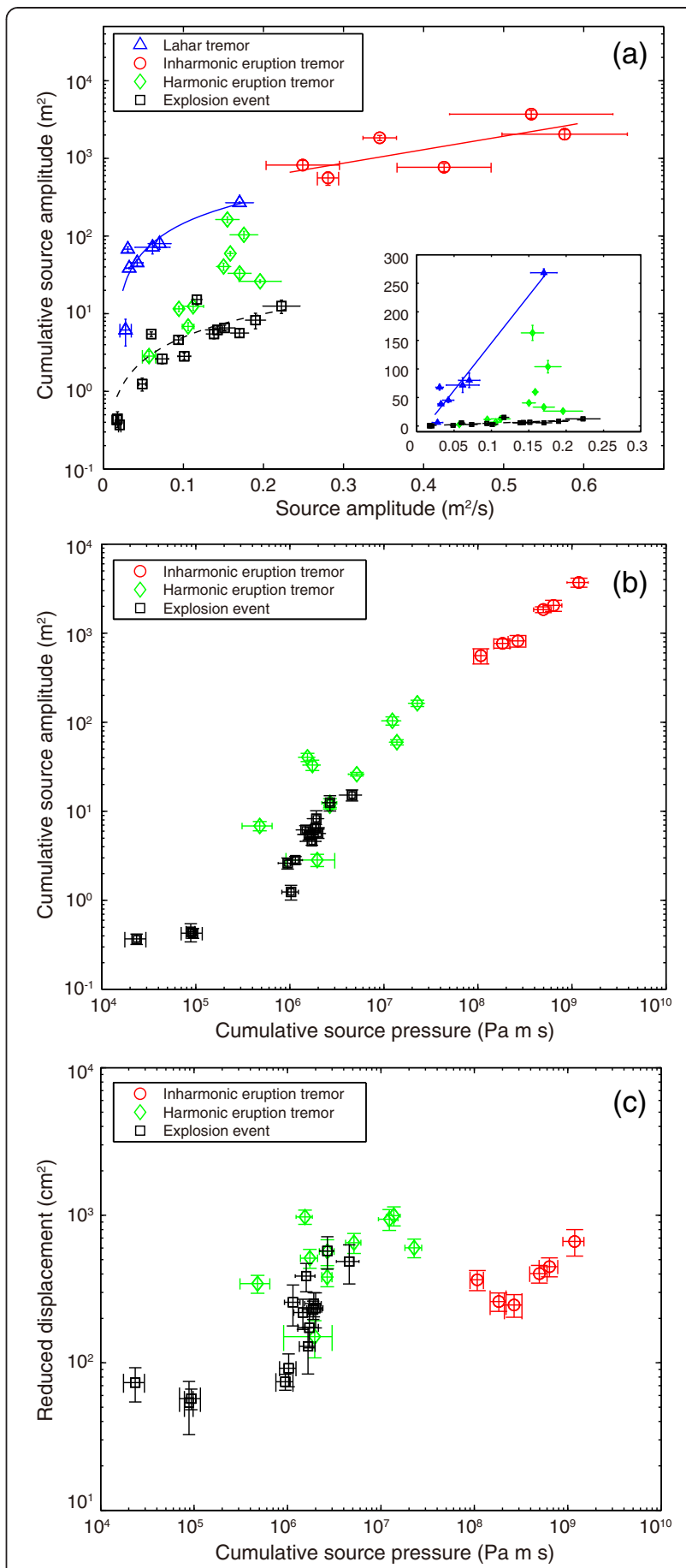

Fig. 5 Comparison of source parameters. a Cumulative source amplitude versus source amplitude. b Cumulative source amplitude versus cumulative source pressure. c Reduced displacement versus cumulative source pressure. Inset in a is an enlargement of a plotted with a linear scale on the vertical axis. Vertical and horizontal bars on the data points indicate probable errors of the individual estimates

spindle shapes. Our ASL analyses of lahar tremor episodes between 2008 and 2014 (Table 2) showed their sources to be on the northern and western flanks of the 


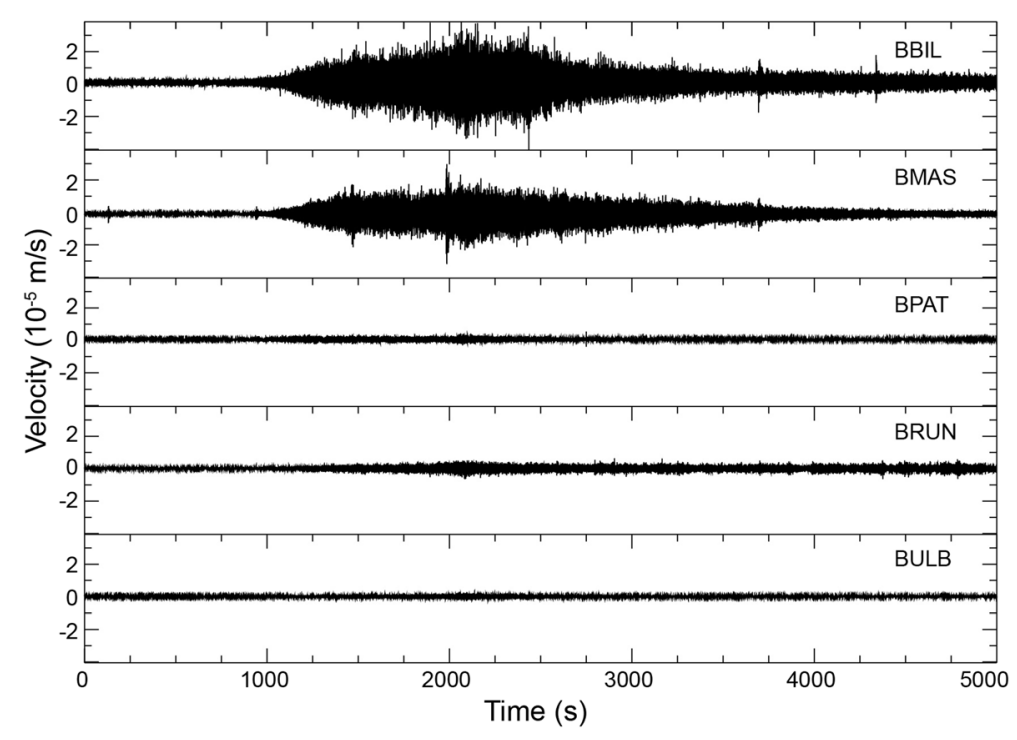

Fig. 6 Vertical unprocessed velocity seismograms of lahar tremor signals on 17 February 2011

volcano (Fig. 1b). Our plot of $I_{\mathrm{s}}$ values versus for lahar tremors (Fig. 5a) suggests that $I_{\mathrm{s}}$ increased linearly with source amplitude.

\section{Discussion}

According to Ichihara et al. (2012), the transfer coefficient for an incident infrasonic wave to generate a seismic wave is on the order of $10^{-7} \mathrm{~m} / \mathrm{s} / \mathrm{Pa}$. The unprocessed seismic velocity and infrasonic data recorded during the eruption episode of 16-17 March 2013 (Fig. 2) have amplitudes of around $10^{-4} \mathrm{~m} / \mathrm{s}$ and $10^{2} \mathrm{~Pa}$, respectively, suggesting that the eruption tremor seismic signals may be affected by infrasonic waves. For explosion events, the seismic waves generated by infrasonic waves were more clearly identified (Fig. 3c). We did not use these to locate the explosion events because the ASLs estimated from them were not near the summit of the volcano. On the other hand, the ASLs of the eruption tremor signals derived from the large amplitude parts of the recorded data were determined to be near the summit (Fig. 1a). This feature suggests that the seismic waves of the eruption tremor signals were dominantly radiated from their sources and the scatters of the ASLs may be caused by the effects of infrasonic waves and other ground noise.

Our results indicate that $I_{\mathrm{s}}$ increases linearly with source amplitude for explosion events and lahar tremor signals, but $I_{\mathrm{s}}$ increases exponentially with source amplitude for inharmonic tremor signals (Fig. 5a). Because the logarithm of the source amplitude is proportional to the magnitude of explosion events (eq. 1), the proportional relationship between source amplitude and $I_{\mathrm{s}}$ for explosion events indicates that $I_{\mathrm{s}}$ can be used as a measure of the explosion event size.

Let us consider the proportional relationship between source amplitude and $I_{\mathrm{s}}$. As shown by eq. $5, I_{\mathrm{s}}$ is the time integration of the source amplitude function, in which the source amplitude represents the maximum amplitude. If we approximate the source amplitude function as a triangular function with a maximum amplitude of $A_{\mathrm{s}}$ and a duration of $T$, we obtain $I_{\mathrm{s}}=A_{\mathrm{s}} T / 2$. Thus, the proportional relationship

Table 2 Lahar tremor episodes analyzed in this study. Tremor duration is not shown because onset and end times were unclear

\begin{tabular}{lll}
\hline Date and time of estimation of source amplitude & Source amplitude $\left(\mathrm{m}^{2} / \mathrm{s}\right)$ & Cumulative source amplitude $\left(\mathrm{m}^{2}\right)$ \\
\hline 2008-08-23 04:58:20 & $(2.78 \pm 0.71) \times 10^{-2}$ & $6.2 \pm 2.3$ \\
2010-05-05 19:27:20 & $(3.24 \pm 0.41) \times 10^{-2}$ & $(3.86 \pm 0.08) \times 10^{1}$ \\
2010-11-16 20:21:10 & $(1.70 \pm 0.18) \times 10^{-1}$ & $(2.69 \pm 0.07) \times 10^{2}$ \\
2011-02-17 05:23:00 & $(7.0 \pm 1.4) \times 10^{-2}$ & $(8.0 \pm 1.3) \times 10^{1}$ \\
2011-11-11 20:56:30 & $(6.2 \pm 2.2) \times 10^{-2}$ & $(7.2 \pm 1.3) \times 10^{1}$ \\
2014-03-11 22:29:00 & $(4.22 \pm 0.75) \times 10^{-2}$ & $(4.52 \pm 0.42) \times 10^{1}$ \\
2014-04-24 16:32:30 & $(3.06 \pm 0.50) \times 10^{-2}$ & $(6.78 \pm 0.49) \times 10^{1}$ \\
\hline
\end{tabular}


between the source amplitude and $I_{\mathrm{s}}$ may occur when $T$ is constant (i.e., if all events have similar durations). We considered the source characteristics of explosion event waveforms recorded at station BBIL (Fig. 7a) for which sources were located near the summit vent. The explosion events had different amplitudes but similar durations (Fig. 7a), indicating that the proportional relation mentioned above holds, thus suggesting that for explosion events, duration is not dependent on source amplitude or magnitude.

It is interesting that the proportional relation between source amplitude and $I_{\mathrm{s}}$ may also hold for lahar tremor signals (Fig. 5a). Six out of seven lahar tremor signals corrected for geometrical spreading and medium attenuation (Fig. 8) were of roughly the same durations
(2000-3000 s) but had different amplitudes; the seventh had the smallest $I_{\mathrm{s}}$ and a much shorter duration. The sources of the lahar tremor signals were located in different regions (Fig. 1b), indicating that our estimated lahar source character was not controlled by local topographic and/or sedimentary conditions.

For the inharmonic tremor signals, $I_{\mathrm{s}}$ increased exponentially with source amplitude (Fig. 5a) and the relation between $I_{\mathrm{s}}$ and $I_{\mathrm{p}}$ was proportional (Fig. $5 \mathrm{~b}$ ). According to the study of Johnson (2003), which was based on the linear theory of sound (Lighthill 1978), $I_{\mathrm{p}}$ defined by eq. 6 may be proportional to the cumulative mass flux or total mass ejected during an eruption, although turbulencerelated processes have also been proposed to explain complex spectral features of infrasonic records observed

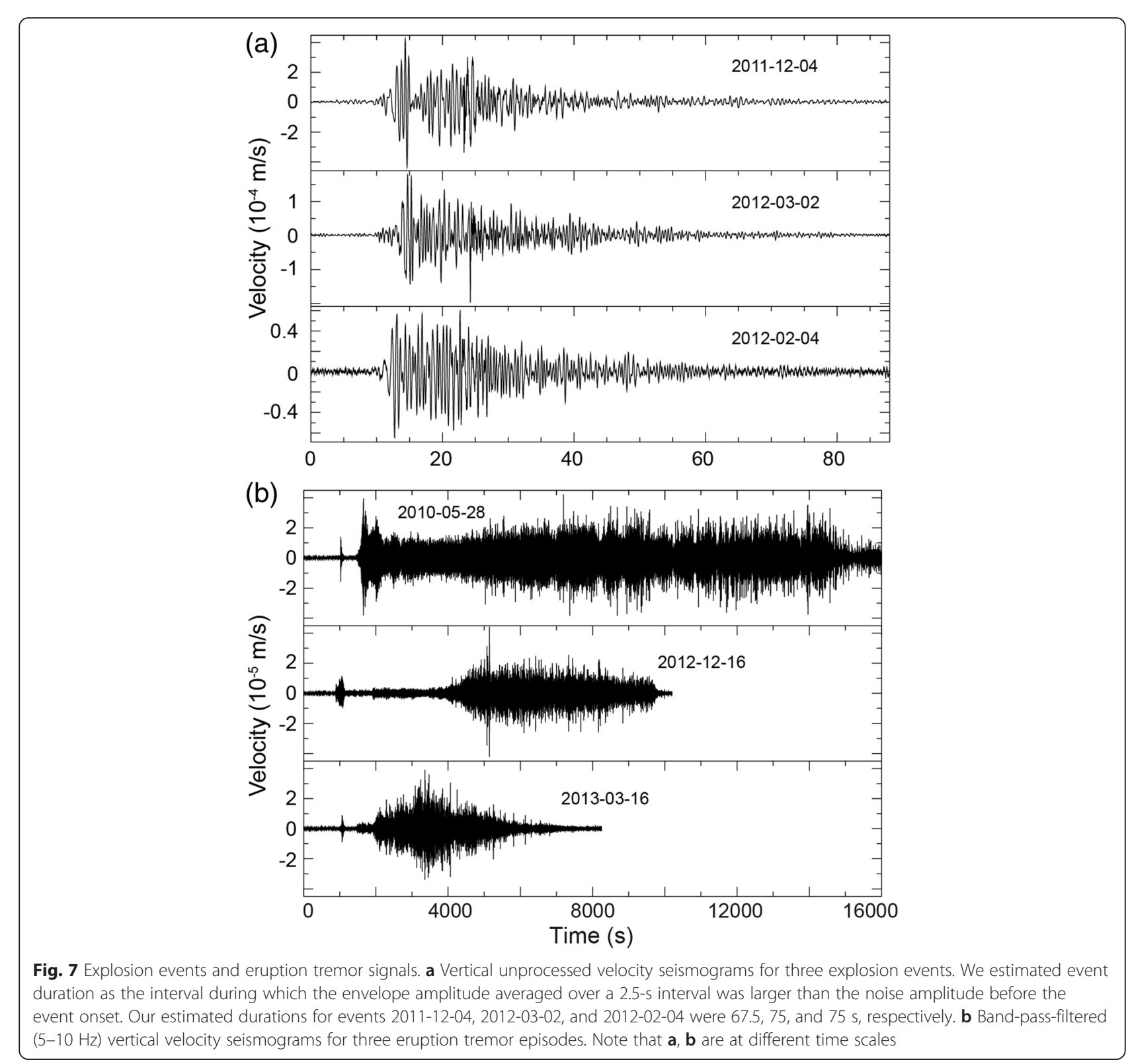




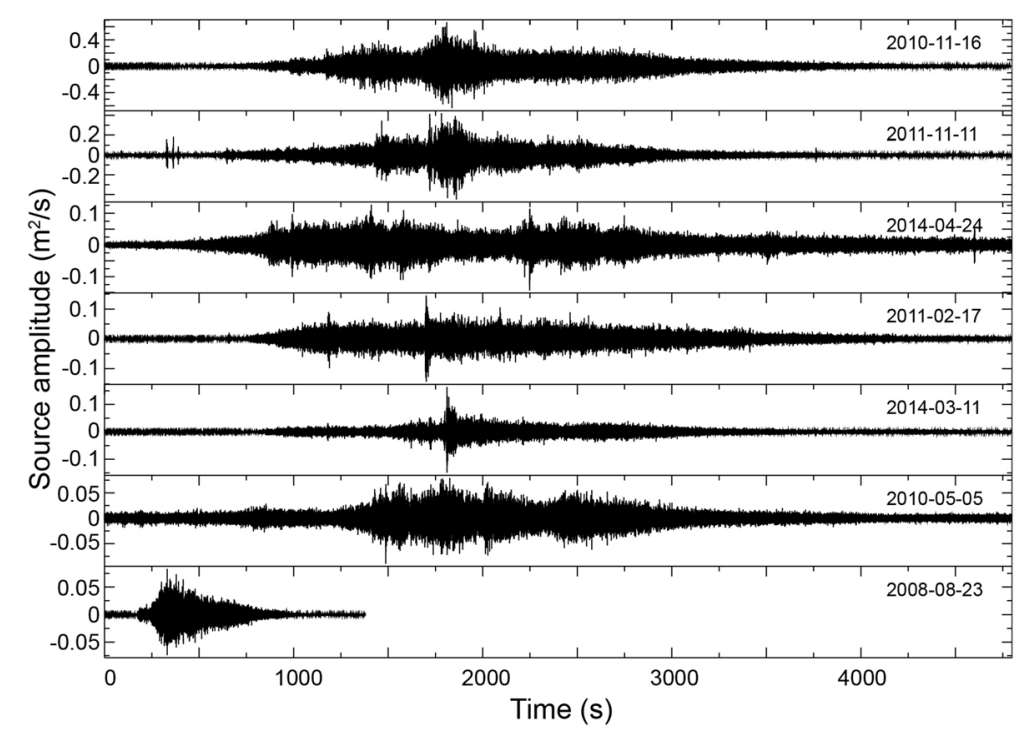

Fig. 8 Lahar tremor signals corrected for geometrical spreading and medium attenuation for seven lahar tremor episodes

during eruptions at Tungurahua (e.g., Fee et al. 2010). The proportional relationship between $I_{\mathrm{s}}$ and $I_{\mathrm{p}}$ suggests that $I_{\mathrm{s}}$ can be used as a measure of the total ejected mass during an eruption and accordingly that the source amplitude may be proportional to the peak mass flux.

The exponential increase of $I_{\mathrm{s}}$ with source amplitude $\left(A_{\mathrm{s}}\right)$ can be given $I_{\mathrm{s}}=\alpha 10^{\beta A_{\mathrm{s}}}$, where $\alpha$ and $\beta$ are constants. This relation suggests that the source amplitude function of an inharmonic eruption tremor has a typical amplitude $(\alpha)$ with duration $(T)$ proportional to the exponential function of the source amplitude $\left(T \propto 10^{\beta A_{s}}\right)$. The inharmonic tremor signals at station BBIL in the $5-10-\mathrm{Hz}$ frequency band (Fig. 7b) have amplitudes around $10^{-5} \mathrm{~m} / \mathrm{s}$ and their durations vary considerably.

The above relation does not hold for harmonic tremor signals (Fig. 5a), although the relation between $I_{\mathrm{s}}$ and $I_{\mathrm{p}}$ is proportional (Fig. 5b). Some of the harmonic tremor signals had similar source amplitudes but different $I_{\mathrm{s}}$ values (Fig. 5a). These signals were characterized by different durations of harmonic oscillation with similar amplitudes.

The proportionality between $I_{\mathrm{p}}$ and $D_{\mathrm{R}}$ is not clear, especially for the tremor signals (Fig. $5 \mathrm{c}$ ). Although the tremor source can be characterized by using both source amplitude and $I_{\mathrm{s}}$, it cannot be characterized by using $D_{\mathrm{R}}$ alone. Thus, to characterize a tremor source, it is important to use a reference frequency band and to take into account the duration of tremor.

To quantify the amounts of tephra fallout at Tungurahua during eruptions in December 2012 and March 2013, Bernard et al. (2013) collected tephra samples at more than 40 locations. They found that the volume of fallout for the December 2012 eruption $\left((2.84-4.59) \times 10^{5} \mathrm{~m}^{3}\right)$ was larger than that of the March 2013 eruption $\left((1.70-2.33) \times 10^{5} \mathrm{~m}^{3}\right)$. For the March 2013 eruption, we observed inharmonic tremor signals and very few harmonic tremor signals during 16-17 March 2013, and we estimated $I_{\mathrm{s}}$ for inharmonic tremor to be $7.68 \times 10^{2} \mathrm{~m}^{2}$ (Table 1). For the December 2012 eruption, we observed inharmonic tremor on 16 December, which was followed by harmonic tremor activity during 17-20 December. The sum of $I_{\mathrm{s}}$ for the inharmonic tremor of 16 December 2012 is $2.66 \times 10^{3} \mathrm{~m}^{2}$ (Table 1 ), which is roughly three times that of 16-17 March 2013. This difference of $I_{\mathrm{s}}$ estimates is roughly consistent with the difference in the volume estimates of Bernard et al. (2013).

We also estimated cumulative $I_{\mathrm{s}}$ during the harmonic tremor activity of 17-20 December 2012 and 16-17 March 2013, for which we assumed a fixed source location at the summit vent. Our estimate of the sum of $I_{\mathrm{s}}$ for all of the inharmonic and harmonic tremor signals during 16-20 December 2012 was $1.76 \times 10^{4} \mathrm{~m}^{2}$, which is 31 times that of $16-17$ March $2013\left(5.64 \times 10^{2} \mathrm{~m}^{2}\right)$ and is clearly inconsistent with the volume estimates of Bernard et al. (2013).

Harmonic tremor signals can be generated by repetitive triggering of a fluid-filled cavity (e.g., Aki et al. 1977; Chouet 1986), self-excited oscillations associated with a fluid flow in a volcanic conduit (Julian 1994), or magma column oscillations (Jellinek and Bercovici 2011; Bercovici et al. 2013). Our results may be explained if we consider that the inharmonic tremor signal is directly related to mass transport associated with magma eruptions and that the harmonic tremor is triggered by a gas flow without tephra fallout. 


\section{Conclusions}

We proposed an approach to characterize sources of tremor signals using the ASL method and applied it to estimate source location, source amplitude, and cumulative source amplitude during tremor at Tungurahua volcano, Ecuador. For eruption tremor, we also estimated the cumulative source pressure from infrasonic records. We estimated these parameters for eruption and lahar tremor signals and explosion events recorded by five broadband seismic and infrasonic stations. We identified two types of eruption tremor, noise-like inharmonic waveforms and harmonic oscillatory signals. We showed that cumulative source amplitude increased linearly with increasing source amplitude for lahar tremor signals and explosion events, but $I_{\mathrm{s}}$ increased exponentially with increasing source amplitude for inharmonic eruption tremor signals. We also found a proportional relation between cumulative source amplitude and cumulative source pressure for the explosion events and eruption tremor, which suggests that cumulative source amplitude may be used as a measure of total ejecta mass during an eruption episode. The cumulative source amplitudes we estimated for inharmonic tremor were consistent with previous estimates of tephra fallout volume. Automated ASL analysis can be applied to estimate source parameters in real time, which would contribute to early assessment of the size of eruptions and lahars. This is of particular importance in rapid hazard evaluations. Future studies will be necessary to test the applicability of the approach described here at other volcanoes and to clarify the variety and universality of the scaling relations among source parameters. These efforts would contribute to a better understanding of the dynamic processes of volcanic eruptions and lahars and lead to improvements in monitoring of volcanic activity and associated seismicity.

\section{Competing interests}

The authors declare that they have no competing interests.

\section{Authors' contributions}

HK developed the main framework of the study, analyzed the data, and drafted the manuscript. PM provided information about lahars and eruptions and revised the manuscript. MR contributed to the interpretation of the seismic data. YM interpreted the scaling relations among source parameters. All authors read and approved the final manuscript.

\section{Acknowledgements}

We thank Mie Ichihara for the useful discussions about infrasonic source processes. We appreciate the comments from two anonymous reviewers and Martha Savage, which helped improve this manuscript.

\section{Author details}

${ }^{1}$ Graduate School of Environmental Studies, Nagoya University, Furo-cho, Chikusa-ku, Nagoya 464-8601, Japan. ${ }^{2}$ Instituto Geofísico, Escuela Politécnica Nacional, P. O. Box 17-01-2759, Quito, Ecuador.

Received: 31 May 2015 Accepted: 26 October 2015

Published online: 04 November 2015

\section{References}

Aki K, Koyanagi R. Deep volcanic tremor and magma ascent mechanism under Kilauea, Hawaii. J Geophys Res. 1981;86:7095-109.

Aki K, Fehler M, Das S. Source mechanism of volcanic tremor: fluid-driven crack models and their application to the 1963 Kilauea eruption. J Volcanol Geotherm Res. 1977:2:259-87.

Alparone S, Andronico D, Lodato L, Sgroi T. Relationship between tremor and volcanic activity during the Southeast Crater eruption on Mount Etna in early 2000. J Geophys Res. 2003;108:2241. doi:10.1029/2002JB001866.

Battaglia J, Aki K. Location of seismic events and eruptive fissures on the Piton de la Fournaise Volcano using seismic amplitudes. J Geophys Res. 2003;108:2364. doi:10.1029/2002JB002193.

Battaglia J, Got JL, Okubo P. Location of long-period events below Kilauea Volcano using seismic amplitudes and accurate relative relocation. J Geophys Res. 2003;108:2553. doi:10.1029/2003JB002517.

Battaglia J, Aki K, Ferrazzini V. Location of tremor sources and estimation of lava output using tremor source amplitude on the Piton de la Fournaise volcano: 1. Location of tremor sources. J Volcanol Geotherm Res. 2005a;147:268-90.

Battaglia J, Aki K, Staudacher T. Location of tremor sources and estimation of lava output using tremor source amplitude on the Piton de la Fournaise volcano: 2. Estimation of lava output. J Volcanol Geotherm Res. 2005b;147:291-308.

Benoit JP, McNutt SR, Barboza V. Duration-amplitude distribution of volcanic tremor. J Geophys Res. 2003;108:2146. doi:10.1029/2001JB001520.

Bercovici D, Jellinek AM, Michaut C, Roman DC, Morse R. Volcanic tremors and magma wagging: gas flux interactions and forcing mechanism. Geophys J Int. 2013;203:1-22. doi:10.1093/gji/ggt277.

Bernard B, Bustillos J, Wade B, Hidalgo S. Influence of the wind direction variability on the quantification of tephra fallouts: December 2012 and March 2013 Tungurahua eruptions. Avances. 2013:5:A14-21.

Chouet B. Dynamics of a fluid-driven crack in three dimensions by the finite difference method. J Geophys Res. 1986;91:13967-92.

Fee D, Garces M, Steffke A. Infrasound from Tungurahua Volcano 2006-2008: Strombolian to Plinian eruptive activity. J Volcanol Geotherm Res. 2010;193:67-81. doi:10.1016/j.jvolgeores.2010.03.006

Fehler M. Observations of volcanic tremor at Mount St. Helens Volcano. J Geophys Res. 1983;88:3476-83.

Garcés MA, Hagerty MT, Schwartz SY. Magma acoustics and time-varying melt properties at Arenal Volcano, Costa Rica. Geophys Res Lett. 1998;25:2293-6. doi:10.1029/98gl01511.

Hall ML, Robin C, Beate B, Mothes P, Monzier M. Tungurahua Volcano, Ecuador: structure, eruptive history and hazards. J Volcanol Geotherm Res. 1999:91:1-21.

Hall ML, Steele AL, Mothes PA, Ruiz MC. Pyroclastic density currents (PDC) of the 16-17 August 2006 eruptions of Tungurahua volcano, Ecuador: Geophysical registry and characteristics. J Volcanol Geotherm Res. 2013;265:78-93. doi:10.1016/j.jvolgeores.2013.08.011

Ichihara M, Takeo M, Yokoo A, Oikawa J, Ohminato T. Monitoring volcanic activity using correlation patterns between infrasound and ground motion. Geophys Res Lett. 2012;39, L04304. doi:10.1029/2011GL050542.

Ichihara M, Lyons J, Yokoo A. Switching from seismic to seismo-acoustic harmonic tremor at a transition of eruptive activity during the Shinmoe-dake 2011 eruption. Earth Planets Space. 2013;65:10. doi:10.5047/eps.2013.05.003.

Jellinek AM, Bercovici D. Seismic tremors and magma wagging during explosive volcanism. Nature. 2011;470:522-5. doi:10.1038/nature09828.

Johnson JB. Generation and propagation of infrasonic airwaves from volcanic explosions. J Volcanol Geotherm Res. 2003;121:1-14.

Julian BR. Volcanic tremor: nonlinear excitation by fluid flow. J Geophys Res. 1994:99:11859-77.

Kumagai H, Nakano N, Maeda T, Yepes H, Palacios P, Ruiz M, et al. Broadband seismic monitoring of active volcanoes using deterministic and stochastic approaches. J Geophys Res. 2010;115, B08303. doi:10.1029/2009JB006889.

Kumagai H, Saito T, O'Brien G, Yamashina T. Characterization of scattered seismic wavefields simulated in heterogeneous media with topography. J Geophys Res. 2011a;116, B03308. doi:10.1029/2009JB007718.

Kumagai H, Palacios P, Ruiz M, Yepes H, Kozono T. Ascending seismic source during an explosive eruption at Tungurahua volcano, Ecuador. Geophys Res Lett. 2011b;38, L01306. doi:10.1029/2010GL045944.

Kumagai $H$, Lacson Jr R, Maeda Y, Figueroa II MS, Yamashina T, Ruiz M, et al. Source amplitudes of volcano-seismic signals determined by the amplitude source location method as a quantitative measure of event size. J Volcanol Geotherm Res. 2013;257:57-71. doi:10.1016/j.jvolgeores.2013.03.002. 
Kumagai H, Lacson Jr R, Maeda Y, Figueroa II MS, Yamashina T. Shallow S wave attenuation and actively degassing magma beneath Taal Volcano, Philippines. Geophys Res Lett. 2014;41:6681-8. doi:10.1002/2014GL061193.

Lees J, Johnson MJB, Ruiz M, Troncoso L, Welsh M. Reventador Volcano 2005: eruptive activity inferred from seismo-acoustic observation. J Volcanol Geotherm Res. 2008;176:179-90. doi:10.1016/j.jvolgeores.2007.10.006.

Lighthill MJ. Waves in fluids. New York: Cambridge University Press; 1978.

Matsumoto S, Shimizu H, Matsushima T, Uehira K, Yamashita Y. Short-term spatial change in a volcanic tremor source during the 2011 Kirishima eruption. Earth Planets Space. 2013;65:323-9. doi:10.5047/eps.2012.09.002.

McNutt SR. Volcanic Tremor. In: Nierenberg WA, editor. Encyclopedia of earth system science, vol. 4. San Diego: Academic Press; 1992. p. 417-25.

McNutt SR. Volcanic tremor amplitude correlated with eruption explosivity and its potential use in determining ash hazards to aviation. US Geological Surv Bull. 1994;2047:377-85.

McNutt SR. Volcanic tremor and its use in estimating eruption parameters. Proceedings of the 2 nd international conference on volcanic ash and aviation safety, U.S. Dept. of Commerce, NOAA, section 2. 2004. p. 49-50.

McNutt SR, Nishimura T. Volcanic tremor during eruptions: temporal characteristics, scaling and constraints on conduit size and processes. J Volcanol Geotherm Res. 2008;178:10-8. doi:10.1016/j.jvolgeores.2008.03.010.

Mothes PA, Yepes HA, Hall MA, Ramón PA, Steele AL, Ruiz MC. The scientific-community interface over the fifteen-year eruptive episode of Tungurahua Volcano, Ecuador. J Appl Volcanol. 2015;4:9. doi:10.1186/ s13617-015-0025-y.

Newhall CG, Self S. The volcanic explosivity index (VEI): an estimate of explosive magnitude for historical volcanism. J Geophys Res. 1982;87:1231-7.

Ogiso M, Yomogida K. Migration of tremor locations before the 2008 eruption of Meakandake volcano, Hokkaido, Japan. J Volcanol Geotherm Res. 2012;217-218:8-20. doi:10.1016/j.jvolgeores.2011.12.005.

Ogiso M, Yomogida K. Estimation of locations and migration of debris flows on Izu-Oshima Island, Japan. J Volcanol Geotherm Res. 2015;298:15-26. doi:10.1016/j.jvolgeores.2015.03.015.

Samaniego P, Le Penec J-L, Robin C, Hidalgo S. Petrological analysis of the pre-eruptive magmatic process prior to the 2006 explosive eruptions at Tungurahua volcano (Ecuador). J Volcanol Geotherm Res. 2011;199:69-84. doi:10.1016/j.jvolgeores.2010.10.010.

Takemura S, Furumura T, Saito T. Distortion of the apparent S-wave radiation pattern in the high-frequency wavefield: Tottri-Ken Seibu, Japan earthquake of 2000. Geophys J Int. 2009;178:950-61. doi:10.111/j.1365-246×2009.04210X.

Watanabe $\mathrm{H}$. Determination of earthquake magnitude at regional distance in and near Japan. Zisin, second series 24. 1971. p. 189-200.

\section{Submit your manuscript to a SpringerOpen ${ }^{\circ}$ journal and benefit from:}

- Convenient online submission

- Rigorous peer review

- Immediate publication on acceptance

- Open access: articles freely available online

- High visibility within the field

- Retaining the copyright to your article

Submit your next manuscript at $>$ springeropen.com 